\title{
Zum Mechanismus der oxidativen Dimerisierung von Aminobenzolen[1]
}

Von F. Effenberger, W. D. Stohrer und A. Steinbach ${ }^{[*]}$

Als Zwischenstufe der oxidativen Dimerisierung $N, N$-disubstituierter Aniline werden Verbindungen des Typs (3) postuliert, die bisher jedoch noch nicht isoliert werden konnten [2].

Bei der Oxidation symmetrischer Triaminobenzole (I) [3] gelang uns jetzt die Darstellung der in kristallinem Zustand roten $\sigma$-Komplexe ( 3 ) [UV-Absorption von ( $3 a$ ) in $\mathrm{C}_{2} \mathrm{H}_{5} \mathrm{OH}$ : $\lambda_{\max }=431 \mathrm{~nm}, \varepsilon=12800 ; \lambda_{\max }=346 \mathrm{~nm}, \varepsilon=41000$ ], die erst bei Einwirkung von Basen die Hexaamino-biphenyle (4) ergeben.

Wir haben die Oxidation mit Silbersalzen in Dimethylformamid und mit Tetrahalogenmethanen, Halogencyanen oder Halogenen in Dichlormethan bei Raumtemperatur durchgeführt. Die Salze (3), deren Struktur durch Analysenwerte, Molekulargewichte und ${ }^{1} \mathrm{H}-\mathrm{NMR}$-Spektren $[(3 b)$ in flüssigem $\mathrm{SO}_{2}: \mathrm{H}_{\alpha}=$ Singulett bei $\tau=5,03, \mathrm{H}_{\beta}=$ Singulett bei $\tau=5,67]$ gesichert ist, fallen dabei in guten Ausbeuten an. Bemerkenswert ist das Auftreten eines stabilen $\mathrm{CBr}_{5}$-Anions bei der Umsetzung von 1,3,5-Tripyrrolidinobenzol mit $\mathrm{CBr}_{4}$ in Dichlormethan. Selbst durch tagelange Extraktion mit Äther wird aus (3a) kein $\mathrm{CBr}_{4}$ freigesetzt. Bei Behandlung mit alkoholischer Natriumperchloratlösung entsteht aus (3a) glatt (3b). Die Struktur von $\mathrm{CBr}_{5} \ominus$ wird gegenwärtig untersucht. 


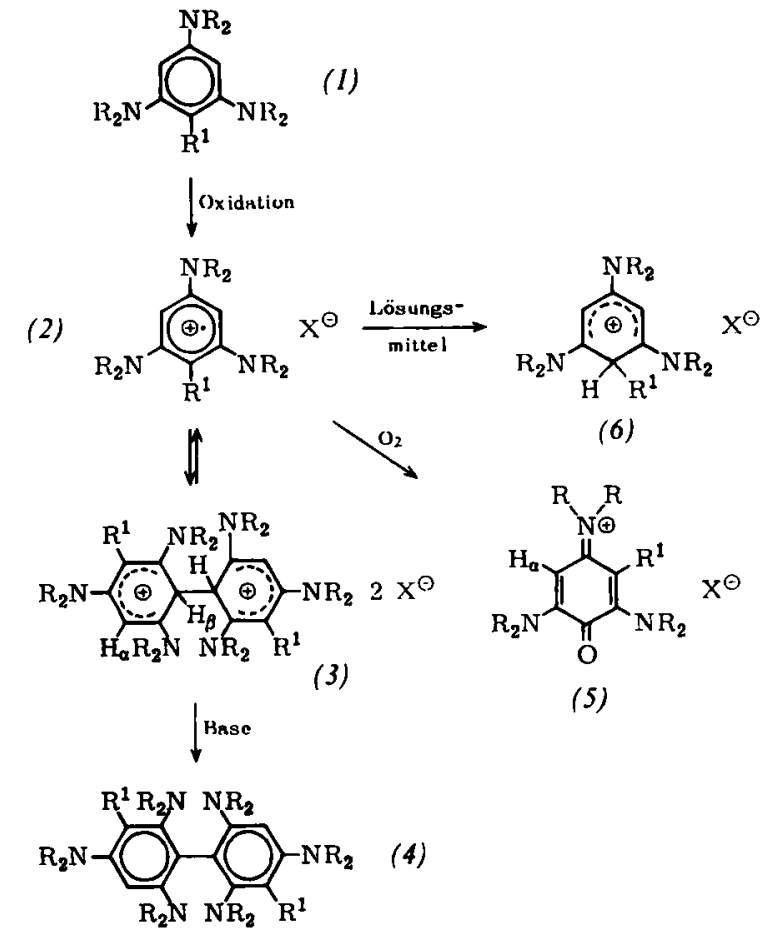

\begin{tabular}{l|l|l|l|l|l|l|l}
\hline & & & & \multicolumn{2}{|c|}{$(3)$} & \multicolumn{2}{|c}{$(5)$} \\
& $-\mathrm{R}-\mathrm{R}-$ & $\mathrm{R}^{1}$ & $\mathrm{X}$ & $\mathrm{Fp}\left({ }^{\circ} \mathrm{C}\right)$ & $\begin{array}{l}\text { Ausb. } \\
(\%)\end{array}$ & $\begin{array}{l}\mathrm{Fp} \\
\left({ }^{\circ} \mathrm{C}\right)\end{array}$ & $\begin{array}{l}\text { Ausb. } \\
(\%)\end{array}$ \\
\hline (a) & $-\left(\mathrm{CH}_{2}\right)_{4}-$ & $\mathrm{H}$ & $\mathrm{CBr}_{5}$ & $177-178$ & 33 & - & - \\
(b) & $-\left(\mathrm{CH}_{2}\right)_{4}-$ & $\mathrm{H}$ & $\mathrm{ClO}_{4}$ & $254-256$ & 91 & 200 bis & 93 \\
& & & & & 201 & \\
(c) & $-\left(\mathrm{CH}_{2}\right)_{4}-$ & $\mathrm{CH}_{3}$ & $\mathrm{ClO}_{4}$ & $158-159$ & 82 & - & - \\
(d) & $-\left(\mathrm{CH}_{2}\right)_{4}-$ & $\mathrm{C}_{2} \mathrm{H}_{5}$ & $\mathrm{ClO}_{4}$ & $123-127$ & 96 & - & - \\
(e) & $-\left(\mathrm{CH}_{2}\right)_{5}-$ & $\mathrm{H}$ & $\mathrm{ClO}_{4}$ & $215-216$ & 56 & - & - \\
(f) & $-\left(\mathrm{CH}_{2}\right)_{2}-\mathrm{O}-\left(\mathrm{CH}_{2}\right)_{2}-$ & $\mathrm{H}$ & $\mathrm{J}_{3}$ & $140-145$ & 2,5 & - & -
\end{tabular}

Die Dimeren (3) dissoziieren beim Erwärmen oder beim Stehenlassen in polaren Lösungsmitteln zu den Radikalkationen (2), die unter H-Abstraktion aus dem Lösungsmittel (6) bilden [4, 5]. Die Neigung zu dissoziieren ist bei den alkylsubstituierten Derivaten (3c) und (3d) wesentlich größer als bei $(3 b)$.

ESR-spektroskopisch können die offensichtlich wenig stabilen Radikalkationen (2) nicht nachgewiesen werden [6]. Daß sie primär entstehen, geht z.B. aus der Bildung eines grünen Salzes hervor, das bei der Oxidation von (1), $\mathrm{R}-\mathrm{R}=\left(\mathrm{CH}_{2}\right)_{4}$, $\mathbf{R}^{1}=\mathbf{H}$, in Anwesenheit von Luftsauerstoff entsteht und dem wir aufgrund der Analyse, dem Molekulargewicht und der spektroskopischen Daten [UV-Absorption in $\mathrm{CH}_{3} \mathrm{OH}$ : $\lambda_{\max }=627 \mathrm{~nm}, \varepsilon=3300 ; \lambda_{\max }=431 \mathrm{~nm}, \varepsilon=10680$; ${ }_{1} \mathrm{H}$-NMR-Spektrum in $\mathrm{CDCl}_{3}: \mathrm{H}_{\alpha}=$ Singulett bei $\tau=4,69$; IR-Absorption: $v(C=O)=1700 \mathrm{~cm}^{-1}$ ] die Struktur (5) zuordnen. Ein chemischer Strukturbeweis für (5) steht noch aus.

Eingegangen am 5. Februar 1969 [Z 947]

[*] Doz. Dr. F. Effenberger, Dipl.-Chem. W. D. Stohrer und Dipl.-Chem. A. Steinbach

Institut für Organische Chemie der Universităt 7000 Stuttgart, Azenbergstraße 14/16

[1] Diese Arbeit wurde von der Deutschen Forschungsgemeinschaft und dem Fonds der Chemischen Industrie gefördert.

[2] E. T. Seo, R. F. Nelson, J. M. Fritsch, L. S. Marcoux, D. W. Leedy u. $R$. N. Adams, J. Amer. chem. Soc. 88, 3498 (1966); $R$. F. Nelson u. R. N. Adams, ibid. 90, 3925 (1968); J. R. Lindsay Smith, R. O. C. Norman u. W. M. Walker, J. chem. Soc. (London) B 1968,$269 ;$ V. Stérba, Z. Ságner u. M. Matrka, Collect. czechoslov. chem. Commun. 30, 2477 (1965).

[3] F. Effenberger u. R. Niess, Chem. Ber. 101, 3787 (1968).

[4] F. Effenberger u. R. Niess, Angew. Chem. 79, 1100 (1967); Angew. Chem. internat. Edit. 6, 1067 (1967).

[5] R. Niess, $K$. Nagel u. F. Effenberger, Tetrahedron Letters $1968,4265$.

[6] Für die ESR-Untersuchungen danken wir Dr. K. Scheffler und Dr. H. Stegmann, Tübingen. 